\title{
Ocular tuberculosis: current perspectives
}

\author{
Faiz I Shakarchi ${ }^{1,2}$ \\ 'Ibn Al-Haetham Teaching \\ Eye Hospital, ${ }^{2}$ Department of \\ Opthalmology, Medical College, \\ Al-Mustansiriya University, \\ Baghdad, Iraq
}

This article was published in the following Dove Press journal:

Clinical Ophthalmology

26 November 2015

Number of times this article has been viewed
Abstract: The World Health Organization currently estimates that nearly two billion people, or one-third of the world's population, are infected by tuberculosis, and that roughly $10 \%$ of the infected people are symptomatic. Tuberculosis affects the lungs in $80 \%$ of patients, while in the remaining $20 \%$ the disease may affect other organs, including the eye. Uveitis can be seen concurrently with tuberculosis, but a direct association is difficult to prove. Ocular tuberculosis is usually not associated with clinical evidence of pulmonary tuberculosis, as up to $60 \%$ of extrapulmonary tuberculosis patients may not have pulmonary disease. The diagnosis of tuberculous uveitis is often problematic and in nearly all reported cases, the diagnosis was only presumptive. Tuberculous uveitis is a great mimicker of various uveitis entities and it can be considered in the differential diagnosis of any type of intraocular inflammation. It is still unknown if ocular manifestations result from a direct mycobacterium infection or hypersensitivity reaction and this is reflected on the management of tuberculous uveitis. Prevalence of tuberculosis as an etiology of uveitis may reach up to $10 \%$ in endemic areas. Tuberculous uveitis is a vision-threatening disease that inevitably leads to blindness if not properly diagnosed and treated. The aim of this review is to illustrate the various clinical features and management of presumed tuberculous uveitis. The current review focuses on the diagnostic criteria, significance of tuberculin skin test, and use of systemic corticosteroids in the management of tuberculous uveitis as recommended in recent publications.

Keywords: tuberculosis, uveitis, choroiditis, tuberculin skin test

\section{Introduction}

Tuberculosis (TB), a multisystem infectious disease caused by Mycobacterium tuberculosis (MTB). TB has existed since ancient times. Evidence of tuberculous pathology was present in fragments of spinal columns from Egyptian mummies. In ancient Greece, the disease was known as consumption, and was uniformly fatal. In 1882, Robert Koch discovered a staining technique to demonstrate MTB. In 1944, streptomycin was first used to treat patients with pulmonary TB. ${ }^{1}$

TB is the leading infectious cause of morbidity and mortality worldwide. According to The World Health Organization, approximately one-third of the world's population, approximately two billion persons, are infected by TB; $10 \%$ of infected people are symptomatic and $90 \%$ have latent TB. ${ }^{2,3}$ Persons with latent MTB infection do not manifest symptoms of active TB and are not infectious, but they may develop clinical disease at anytime during their life.

More than $95 \%$ of new infections occur in the developing world, particularly in Africa and South Asia. There is an increasing number of TB infections in both the developing and developed world due to multidrug-resistant $\mathrm{TB}$, human immunodeficiency virus (HIV), and global migration. ${ }^{4-6}$ Poor socioeconomic conditions, immunosuppression, and general debility are important predisposing factors irrespective of ethnic origin. $^{7}$
Correspondence: Faiz I Shakarchi Department of Ophthalmology, Medical College, Al-Mustansiriya University, Jinub street, Baghdad, Iraq Email faizalshkr@yahoo.com 
TB is a multisystem disease that primarily affects the lungs and may affect other organs including the eye. Uveitis is the most common ocular manifestation of the disease, but a direct association is difficult to prove. It is still unknown if tuberculous uveitis results from a direct mycobacterium infection or hypersensitivity reaction, and this is reflected on its management.

The aim of this review article is to illustrate the various clinical features and management of presumed tuberculous uveitis. The current review focuses on the diagnostic criteria, significance of tuberculin skin test (TST), and use of systemic corticosteroids in the management of tuberculous uveitis as recommended in recent publications.

\section{Clinical features}

MTB is an obligate aerobic bacteria, usually found in highly oxygenated tissue. TB affects the lungs in $80 \%$ of patients, while in the remaining $20 \%$ the disease may affect other organs, including the eye, in which the choroid has one of the highest oxygen tension in the body.

Ocular TB is defined as an infection by MTB in the eye, around the eye, or on its surface. ${ }^{1}$ Ocular TB is usually not associated with clinical evidence of pulmonary $\mathrm{TB}$, as up to $60 \%$ of extrapulmonary TB patients may not have pulmonary TB. ${ }^{8}$

Ocular TB presents a complex clinical problem due to a wide spectrum of presentations and difficulty in diagnosis. ${ }^{9}$ Ocular TB is either primary in which the eye is the primary port of entry of the mycobacterium into the body, or secondary as a result of seeding by hematogenous spread from a distant site. Primary disease is rare, and includes eyelid, conjunctival, corneal, and scleral lesions, while the uveal tract, retina, and optic nerve are involved in secondary disease. Inflammation of the uveal tract is the most common eye manifestation of the disease, due to its high blood supply. ${ }^{10,11}$

Till before 50 years, TB was considered as the most common cause of granulomatous uveitis, but there has been a dramatic change in the prevalence of ocular TB over the subsequent decades as many of previously unknown etiologies, such as sarcoid, toxoplasmosis, and histoplasmosis, were now recognized. ${ }^{1}$

In recent years, there has been a renewed interest in TB stimulated by a rising incidence, the spread of the HIV pandemic and the emergence of multidrug-resistant strains. ${ }^{9}$

Ocular TB is still an important cause of uveitis. Prevalence of TB as an etiology of uveitis has varied from $0.5 \%$ in the USA, ${ }^{12} 4 \%$ in the People's Republic of China, ${ }^{13} 6.31 \%$ in Italy, ${ }^{14} 6.9 \%$ in Japan, ${ }^{15} 9.86 \%$ in north India, ${ }^{16} 10.5 \%$ in Saudi Arabia, ${ }^{17}$ and $11.4 \%$ in Iraq where TB is endemic. ${ }^{18}$
Intraocular TB is a great mimicker of various uveitis entities and it can be considered in the differential diagnosis of any type of intraocular inflammation. Ocular inflammation could be unilateral or bilateral, sometimes inflammation of one eye starts months or years before the other. ${ }^{19}$ Uveitis can present as anterior, intermediate, posterior, or panuveitis.

Tuberculous anterior uveitis has an insidious onset and runs a chronic course. Anterior uveitis presents as unilateral or bilateral chronic granulomatous disease that manifests as granulomatous keratic precipitates associated sometimes with iris nodules or granulomas. Anterior uveitis is often accompanied by vitritis, and inevitably complicated by the development of posterior synechiae and cataract. ${ }^{1,20}$

The vitreous may be the primary site of inflammation and presents with moderate-to-severe cellular reaction in the vitreous cavity, including snowball opacities. Intermediate uveitis is often associated with granulomatous keratic precipitates. Peripheral retinal vasculitis associated with discrete perivascular choroiditis or scars may indicate a tuberculous etiology. Cystoid macular edema, cataract, peripheral neovascularization, and vitreous hemorrhage can occur with $\mathrm{TB}$ intermediate uveitis..$^{20,21}$

Posterior uveitis is the most common presentation of intraocular TB, with lesions predominantly present in the choroid as focal, multifocal or serpiginous choroiditis, solitary or multiple choroidal nodules (tubercles), choroidal granuloma (tuberculoma), neuroretinitis, subretinal abscess, endophthalmitis, panophthalmitis, and retinal vasculitis, which is frequently ischemic in nature and may lead to proliferative vascular retinopathy with recurrent vitreous hemorrhage, rubeosis iridis, and neovascular glaucoma. ${ }^{19,22-24}$

In a study from India of 158 patients with presumed intraocular TB, 66 (42\%) had posterior uveitis, 57 (36\%) anterior uveitis, 18 (11\%) panuveitis, and the remaining $17(11 \%)$ had intermediate uveitis. ${ }^{25}$ In a study conducted in Saudi Arabia of 51 patients (73 eyes) with presumed tuberculous uveitis, 58 (79.5\%) eyes had panuveitis, and 15 (20.5\%) eyes had posterior uveitis at presentation. Clinical manifestations included vitritis in $52(71.2 \%)$ eyes, macular edema in $46(63 \%)$ eyes, retinal periphlebitis in $26(35.6 \%)$ eyes, multifocal choroiditis in 15 (20.5\%) eyes, and granulomatous anterior uveitis in 13 (17.9\%) eyes. ${ }^{26}$ According to a study ${ }^{19}$ conducted in Iraq that included 64 patients (126 eyes) with presumed TB uveitis; 116 eyes (92.1\%) had panuveitis, six eyes (4.7\%) had posterior uveitis, and four eyes (3.2\%) had intermediate uveitis. Vitritis was a universal finding, while multifocal choroiditis was recorded in 104 eyes $(82.5 \%)$. 
The physical findings mentioned above are suggestive but nonspecific. It is still unknown if ocular manifestations result from a direct mycobacterial infection or a hypersensitivity response to mycobacteria and this is reflected on the management of TB uveitis. The choroidal nodules may suggest direct hematogenous infection while the vasculitis and choroiditis are more likely to be the result of immune hypersensitivity. ${ }^{10,22,27}$

In one study, 50 patients presented with multifocal choroiditis and were treated with antituberculous therapy (ATT) without concomitant use of systemic corticosteroids. All patients treated had a favorable response, and no recurrence was recorded. These findings may indicate that ocular manifestations in these patients were probably due to direct mycobacterial invasion. ${ }^{19}$

\section{Diagnosis}

The diagnosis of ocular TB is often problematic due to a wide spectrum of presentations and it is impractical to take uveal biopsy for culture and direct histopathological examination to provide definitive proof of ocular infection. ${ }^{11} \mathrm{In}$ nearly all reported cases, the diagnosis of ocular TB was only presumptive.

Most patients with ocular involvement have no history of pulmonary or other systemic forms. ${ }^{28}$ The absence of clinically evident pulmonary TB does not rule out the possibility of ocular TB, as $\sim 60 \%$ of patients with extrapulmonary TB have no evidence of pulmonary TB and chest X-rays are normal in cases of latent TB..$^{21,28,29}$

In most studies, the diagnostic criteria for presumed tuberculous uveitis were: residence or migration from areas endemic in TB, previous history of contact with TB-infected patients, presence of suggestive ocular findings, exclusion of other known causes of uveitis, corroborative evidence such as a positive TST, positive interferon-gamma release assays (IGRAs), and a positive response to conventional ATT without recurrence. An extraocular evidence of TB in a patient with uveitis also aids in diagnosing intraocular TB. ${ }^{11,19,21,29}$ In a study that included 64 patients with presumed TB uveitis, 24 patients $(37.5 \%)$ reported that they had previous contact with pulmonary TB-infected patients, sometimes that contact was several years before eye symptoms start. ${ }^{19}$

\section{Tuberculin skin testing}

The TST or the Mantoux test has been used for several decades to detect latent TB. The standard test consists of an intradermal injection of five units of purified protein derivative. An induration of $10 \mathrm{~mm}$ or more after $48-72$ hours is considered positive. However, in patients with HIV infection and those who are immunosuppressed, an induration of 5-10 $\mathrm{mm}$ is taken as positive. An induration of less than $5 \mathrm{~mm}$ is considered to be a negative result. The disease may be associated with false-negative reaction, especially in elderly people, the malnourished, and the immunosuppressed. ${ }^{9}$

False-positive TST can occur in exposure to nontuberculous mycobacteria ${ }^{30}$ and some patients have already received Bacille Calmette-Guerin (BCG), but the effect of BCG on TST declines over the first 7 years after vaccination and strongly positive TST is unlikely to be due to prior BCG vaccination..$^{31} \mathrm{TST}$ can give false-positive result in patients with exaggerated skin hypersensitivity like in Behçet's disease, as it may act as a pathergy test. ${ }^{19}$

Guidelines for interpreting of TST vary in different countries where different strengths are used. The predictive value varies depending on the incidence of TB in the population and local BCG vaccination policy. In the USA, the routine use of TB skin testing in patients with uveitis is considered unhelpful, ${ }^{32}$ whereas in India it is considered mandatory. ${ }^{33}$ A previous study conducted in Iraq, showed a high sensitivity and specificity of strongly positive TST (more than $14 \mathrm{~mm}$ area of induration/necrosis) for ocular TB in the Iraqi population. ${ }^{18}$

\section{Interferon-gamma release assay}

IGRA is based on gamma interferon production by $\mathrm{T}$ cells sensitized to specific antigens, which are specific to MTB and therefore not influenced by BCG and most nontuberculous bacteria. These tests include QuantiFERON-TB Gold In-Tube (QFT; Cellestis Inc., Carnegie, VIC, Australia) and ELISpotPLUS (T-SPOT.TB, Oxford Immunotec, Oxford, UK). The IGRA is approved by the US Food and Drug Administration and many other countries. The T-SPOT.TB test is approved in Europe, it is an enzyme-linked immunospot (ELISpot) assay-based test. ${ }^{34-36}$

IGRAs such as T-SPOT.TB (Oxford Immunotec) and QFT (Cellestis Inc.) are more specific and sensitive than TST in detecting active pulmonary TB infections. ${ }^{37}$ However, they are less sensitive for diagnosing latent TB infections. ${ }^{38}$ T-SPOT.TB is more specific for diagnosing TB-associated uveitis, and serves as a better diagnostic tool if used in conjunction with the TST. ${ }^{39}$ The accuracy of diagnosing TB uveitis increases when both tests are used in combination with suggestive clinical signs. ${ }^{39}$

\section{Molecular techniques}

Polymerase chain reaction techniques were used for the detection of MTB in aqueous and vitreous samples from 
patients with presumed tuberculous uveitis. Detection of antibodies against purified cord factor, the most antigenic and abundant cell wall component of MTB, can provide strong evidence of the infection. ${ }^{40}$ However, the sensitivity was reported to be low, as many ocular manifestations may represent a delayed hypersensitivity reaction rather than a direct mycobacterial infection, making the analysis of a fluid sample from the eye less sensitive. ${ }^{11,24,41}$

\section{Treatment}

The diagnosis of ocular TB is presumptive, and it is unknown if ocular manifestations result from a delayed hypersensitivity reaction or due to the infectious agent. ${ }^{33}$ This is reflected in the absence of information on ocular TB management in any of the TB guidelines of the UK, USA, or Canada. ${ }^{42}$

According to the recommendations of the American Thoracic Society, the Centers for Disease Control and Prevention and the Infectious Diseases Society of America for pulmonary and extrapulmonary TB, four drugs (isoniazid $5 \mathrm{mg} / \mathrm{kg} /$ day, rifampicin $450 \mathrm{mg} /$ day if body weight is $<50 \mathrm{~kg}$ and $600 \mathrm{mg}$ if the weight is $>50 \mathrm{~kg}$, ethambutol $15 \mathrm{mg} / \mathrm{kg} /$ day, and pyrazinamide $25-30 \mathrm{mg} / \mathrm{kg} /$ day) are prescribed initially for 8 weeks, followed by two drugs (rifampicin and isoniazid) for at least another 18 weeks. ${ }^{43}$

The use of oral steroids in patients with presumed tuberculous uveitis is clearly a confounding issue. ${ }^{33}$ Patients treated only with systemic corticosteroids showed worsening or recurrence of the inflammation. ${ }^{23,44}$ Several studies reported a favorable response to ATT when administered concomitantly with systemic corticosteroids in patients with presumed tuberculous uveitis. ${ }^{23,25,44}$ Oral prednisone can be used in the treatment of ocular TB, in order to control the coexisting inflammatory reaction, and reduce macular edema. ${ }^{45}$ It might be desirable diagnostically to delay steroid treatment in order to assess the response to ATT, this must be balanced against the risk of loss of sight. ${ }^{9}$

In a previous study that included 64 patients (126 eyes) with presumed TB uveitis, 50 patients (100 eyes) were treated with ATT drugs only, while systemic corticosteroids (oral prednisone) were added in 14 patients (26 eyes) to decrease macular edema and macular scarring. ${ }^{19}$ All patients treated with ATT drugs only had favorable response and no recurrence was recorded for more than 6 months after completion of treatment. Use of ATT in these patients could help by killing the intraocular microorganisms; thus eliminating the antigen load, the recurrences, and the resultant hypersensitivity inflammation. ${ }^{41}$ Probably, in eyes with presumed TB uveitis, oral prednisone can be added with ATT, only when lesions are involving or threatening the macula in order to decrease macular scarring.

Secondary cataract in eyes with TB uveitis can be safely managed, after controlling of the inflammation, by phacoemulsification with intraocular lens implantation. ${ }^{19}$

\section{Disclosure}

The author reports no conflicts of interest in this work.

\section{References}

1. Samson MC, Foster CS. Tuberculosis. In: Foster CS, Vitale AT, editors. Diagnosis and Treatment of Uveitis. Philadelphia: WB Saunders Company; 2002:264-272.

2. Schlossberg D, Maher D, Raviglione MC. The global epidemic of tuberculosis: a World Health Organization perspective. In: Schlossberg D, editor. Tuberculosis and Nontuberculous Mycobacterial Infections. 4th ed. Chapter 10. Philadelphia: WB Saunders Company; 1999:104-115.

3. Dye C, Scheele S, Dolin P, Pathania V, Raviglione MC. Consensus statement. Global burden of tuberculosis: estimated incidence, prevalence, and mortality by country. WHO Global Surveillance and Monitoring Project. JAMA. 1999;282:677-686.

4. WHO global tuberculosis control: key findings from the December 2009 WHO report. Wkly Epidemiol Rec. 2010;85(9):69-80.

5. Blumberg HM, Migliori GB, Ponomarenko O, Heldal E. Tuberculosis on the move. Lancet. 2010;375(9732):2127-2129.

6. Gandhi NR, Nunn P, Dheda K, et al. Multidrug-resistant and extensively drug-resistant tuberculosis: a threat to global control of tuberculosis. Lancet. 2010;375(9728):1830-1843.

7. Hawker JI, Bakshi S, Ali S, Farrington CP. Ecological analysis of ethnic differences in relation between tuberculosis and poverty. $B M J$. 1999;319:1031-1034.

8. Alvarez S, McCabe WR. Extrapulmonary tuberculosis revisited: a review of experience at Boston City and other hospitals. Medicine (Baltimore). 1984;63(1):25-55.

9. Bodaghi B, LeHoang P. Ocular tuberculosis. Curr Opin Ophthalmol. 2000;11:443-448.

10. Helm CJ, Holland GN. Ocular tuberculosis. Surv Ophthalmol. 1993; 38:229-256.

11. Varma D, Anand S, Reddy AR, et al. Tuberculosis: an under-diagnosed aetiological agent in uveitis with an effective treatment. Eye (Lond). 2006;20:1068-1073.

12. Henderly DE, Genstler AJ, Smith RE, Rao NA. Changing patterns of uveitis. Am J Ophthalmol. 1987;103(2):131-136.

13. Abrahams IW, Jiang YQ. Ophthalmology in China. Endogenous uveitis in a Chinese ophthalmological clinic. Arch Ophthalmol. 1986; 104(3):444-446.

14. Mercanti A, Parolini B, Bonora A, Lequaglie Q, Tomazzoli L. Epidemiology of endogenous uveitis in north-eastern Italy. Analysis of 655 new cases. Acta Ophthalmol Scand. 2001;79(1):64-68.

15. Wakabayashi T, Morimura Y, Miyamoto Y, Okada AA. Changing patterns of intraocular inflammatory disease in Japan. Ocul Immunol Inflamm. 2003;11(4):277-286.

16. Singh R, Gupta V, Gupta A. Pattern of uveitis in a referral eye clinic in north India. Indian J Ophthalmol. 2004;52(2):121-125.

17. Al-Mezaine HS, Kangave D, Abu El-Asrar AM. Patterns of uveitis in patients admitted to a University Hospital in Riyadh, Saudi Arabia. Ocul Immunol Inflamm. 2010;18(6):424-431.

18. Al-Shakarchi FI. Pattern of uveitis at a referral center in Iraq. Middle East Afr J Ophthalmol. 2014;21:291-295.

19. Al-Shakarchi F. Mode of presentations and management of presumed tuberculous uveitis at a referral center. Iraqi Postgrad Med J. 2015;14(1):91-95. 
20. Gupta A, Bansal R, Gupta V, Sharma A, Bambery P. Ocular signs predictive of tubercular uveitis. Am J Ophthalmol. 2010;149(4): 562-570.

21. Parchand S, Tandan M, Gupta V, Gupta A. Intermediate uveitis in Indian population. J Ophthalmic Inflamm Infect. 2011;1(2):65-70.

22. Sheu SJ, Shyu JS, Chen LM, Chen YY, Chirn SC, Wang JS. Ocular manifestations of tuberculosis. Ophthalmology. 2001;108:1580-1585.

23. Gupta V, Gupta A, Arora S, Bambery P, Dogra MR, Agarwal A. Presumed tubercular serpiginous like choroiditis: clinical presentation and management. Ophthalmology. 2003;110:1744-1749.

24. Ishihara M, Ohno S. [Ocular tuberculosis]. Nippon Rinsho. 1998;56: 3157-3161.

25. Gupta V, Gupta A, Rao NA. Intraocular tuberculosis: an update. Surv Ophthalmol. 2007;52:561-587.

26. Al-Mezaine HS, Al-Muammar A, Kangave D, Abu El-Asrar AM. Clinical and optical coherence tomographic findings and outcome of treatment in patients with presumed tuberculous uveitis. Int Ophthalmol. 2008;28:413-423.

27. Sharma A, Thapa B, Lavaju P. Ocular tuberculosis: an update. Nepal J Ophthalmol. 2011;3(5): 52-67.

28. Abu El-Asrar AM, Abouammoh M, Al-Mezaine HS. Tuberculous uveitis. Middle East Afr J Ophthalmol. 2009;16(4):188-201.

29. Cimino L, Herbort CP, Aldigeri R, Salvarani C, Boiardi L. Tuberculous uveitis: a resurgent and underdiagnosed disease. Int Ophthalmol. 2009;29(2):67-74

30. Bruins J, Gribnau JH, Bwire R. Investigation into typical and atypical tuberculin sensitivity in the Royal Netherlands Army, resulting in a more rational indication for isoniazid prophylaxis. Tuber Lung Dis. 1995;76:540-544.

31. Rowland K, Guthmann R, Jamieson B, Malloy D. Clinical inquiries How should we manage a patient with positive PPD and prior BCG vaccination. J Fam Pract. 2006;55:718-720.

32. Rosenbaum JT, Wernick R. The utility of routine screening of patients with uveitis for systemic lupus erythematosus or tuberculosis: a Bayesian analysis. Arch Ophthalmol. 1990;108:1291-1293.

33. Rao NA, Saraswathy S, Smith RE. Tuberculous uveitis: distribution of mycobacterium tuberculosis in the retinal pigment epithelium. Arch Ophthalmol. 2006;124:1777-1779.

34. Moru T, Sakatani M, Yamagishi F, et al. Specific detection of tuberculosis infection: an interferon-based assay using new antigens. Am J Respir Crit Care Med. 2004;170:59-64.
35. Ewer K, Deeks J, Alvarez L, et al. Comparison of T-cell-based assay with tuberculin skin test for diagnosis of Mycobacterium tuberculosis infection in a school tuberculosis outbreak. Lancet. 2003;361:1168-1173.

36. Mazurek GH, Jereb J, Lobue P, Iademarco MF, Metchock B, Vernon A; Division of Tuberculosis Elimination, National Center for HIV, STD, and TB Prevention, Centers for Disease Control and Prevention (CDC). Guidelines for using the QuantiFERON-TB Gold test for detecting Mycobacterium tuberculosis infection, United States. MMWR Recomm Rep. 2005;54(RR-15):49-55.

37. Leung CC, Yam WC, Yew WW, et al. T-Spot.TB outperforms tuberculin skin test in predicting tuberculosis disease. Am J Respir Crit Care Med. 2010;182(6):834-840

38. Kleinert S, Kurzai O, Elias J, et al. Comparison of two interferon-gamma release assays and tuberculin skin test for detecting latent tuberculosis in patients with immune-mediated inflammatory diseases. Ann Rheum Dis. 2010;69(4):782-784.

39. Ang M, Wong W, Ngan CC, Chee SP. Interferon-gamma release assay as a diagnostic test for tuberculosis-associated uveitis. Eye. 2012 26(5):658-665.

40. Sakai JI, Matsuzawa S, Usui M, Yano I. New diagnostic approach for ocular tuberculosis by ELISA using the cord factor as antigen. $\mathrm{BrJ}$ Ophthalmol. 2001;85:130-133.

41. Gupta V, Arora S, Gupta A, Ram J, Bambery P, Sehgal S. Management of presumed intraocular tuberculosis: possible role of the polymerase chain reaction. Acta Ophthalmol Scand. 1998;35:237-239.

42. Sanghvi C, Bell C, Woodhead M, Hardy C, Jones N. Presumed tuberculous uveitis: diagnosis, management, and outcome. Eye (Lond). 2011;25: 475-480.

43. American Thoracic Society, CDC and Infectious Diseases Society of America. Treatment of tuberculosis. MMWR Recomm. Rep. 2003 ; 52:1-77.

44. Morimura Y, Okada AA, Kawahara S, et al. Tuberculin skin testing in uveitis patients and treatment of presumed intraocular tuberculosis in Japan. Ophthalmology. 2002;109:851-857.

45. Alvarez GG, Roth VR, Hodge W. Ocular tuberculosis: diagnostic and treatment challenges. Int J Infect Dis. 2009;13:432-435.
Clinical Ophthalmology

\section{Publish your work in this journal}

Clinical Ophthalmology is an international, peer-reviewed journal covering all subspecialties within ophthalmology. Key topics include: Optometry; Visual science; Pharmacology and drug therapy in eye diseases; Basic Sciences; Primary and Secondary eye care; Patient Safety and Quality of Care Improvements. This journal is indexed on Submit your manuscript here: http://www.dovepress.com/clinical-ophthalmology-journal

\section{Dovepress}

PubMed Central and CAS, and is the official journal of The Society of Clinical Ophthalmology (SCO). The manuscript management system is completely online and includes a very quick and fair peer-review system, which is all easy to use. Visit http://www.dovepress.com/ testimonials.php to read real quotes from published authors. 